\title{
Cervix Cancer Brachytherapy: Target Volume Determination
}

\author{
Melis GÜLTEKIN
}

Department of Radiation Oncology, Hacettepe University, Faculty of Medicine, Ankara-Turkey

\begin{abstract}
SUMMARY
Concurrent chemoradiotherapy followed by brachytherapy (BRT) is the standard treatment for patients with locally advanced cervical cancer. Today, three-dimensional (3D) image-guided BRT (3D-IGBT) is the new standard. It improves local control, increases overall survival, and minimizes toxicity. Magnetic resonance imaging (MRI), ultrasound (US), computed tomography (CT), and positron emission tomography (PET)/CT can be performed in 3D-IGBT. In cervical cancers, MRI is considered the gold standard imaging modality. It also has been implemented into the cervix 3D-IGBT because of the excellent soft tissue contrast with clear definition of target volumes and easily identified organs at risk (OARs). This review summarizes imaging and volume definitions in 3D-IGBT of cervical cancer.

Keywords: Brachytherapy; cervix carcinoma; contouring; imaging; target volume.

Copyright $\odot$ 2019, Turkish Society for Radiation Oncology
\end{abstract}

\section{Introduction}

External beam radiotherapy (EBRT) with concurrent cisplatin-based chemotherapy followed by brachytherapy (BRT) is the standard treatment for patients with locally advanced cervical cancer.[1-3] Dose escalation and greater sparing of the surrounding organs at risk (OARs) can be achieved by using BRT technique, resulting in increased survival rates and reduced toxicity. [4] According to the Surveillance, Epidemiology, and End Results database, a significant survival advantage was observed in patients treated with EBRT and BRT compared with that in EBRT alone.[5]

Based on the improved dosimetric parameters and clinical outcomes in several studies, three-dimensional (3D) image-guided BRT (3D-IGBT) became the new standard in cervical cancer.[6-10] Target volumes and OARs can be delineated more accurately with 3DIGBT. It accounts for the changes in tumor configuration during treatment or the changes in the position of the OARs as a result in the changing tumoral topography. Considering the volume and location of the tu- mor, dose can be increased in large residual tumors or more OARs can be protected in small residual tumors. Thus, local control and survival rates are improved, and morbidity is decreased.[6,11,12] However, 3DIGBT requires a high level of experience, and it is often time consuming and expensive.

3D-IGBT in cervical cancer follows a standard sixstep process: sedation and analgesia, pelvic examination and applicator insertion, imaging, contouring of target volumes and OARs, applicator reconstruction, and treatment planning and plan evaluation. This article summarizes imaging and volume definitions in 3DIGBT of cervical cancer.

\section{Imaging in Cervical Cancer}

In cervical cancer, magnetic resonance imaging (MRI), ultrasound (US), computed tomography (CT), and positron emission tomography (PET)/CT are preferred imaging modalities.

In the evaluation of cervical cancers, MRI is considered the gold standard imaging modality. Tumor size and configuration have been proven to be more 
appropriately assessed by MRI than any other imaging procedure. It is used for staging, treatment planning, monitoring of treatment response, and follow-up. Better image quality; excellent soft tissue contrast; and better uterine, para-uterine tissues, cervix, and tumor differentiation are the most important advantages compared to CT.[13,14] MRI can also be used to perform functional imaging. Diffusion-weighted (DW) MRI has been applied to evaluate the cellular density and membrane integrity. Dynamic contrast-enhanced (DCE) MRI has been applied to evaluate the tumor microvasculature and perfusion (hypoxia).[14-16]

In particular, MRI has been implemented into the cervix 3D-IGBT procedure because of the excellent soft tissue contrast with clear definition of target volumes and easily identified OARs (Fig. 1). We can also assess the adequacy of the application and the presence of perforation.[17] All patients with cervical cancer should undergo MRI at diagnosis and at least just before the first fraction of BRT in addition to clinical examination for treatment planning.[17] Most commonly, 3T MRI is used for diagnostic imaging, and 0.2-1.5 T MRI is used for BRT planning. Multiplanar (transvers, sagittal, coronal, and oblique image orientation) T2-weighted (T2W) images with pelvic surface coils have been considered as the gold standard for delineating the topography of the tumor and the OARs. The use of an intracavitary coil is not recommended because it alters normal pelvic anatomy. Bowel preparation is optional prior to the MRI imaging. However, antiperistaltic agents, such as glucagon, are commonly used to minimize artifacts from bowel movements in BRT planning. [13] Vaginal contrast (e.g. US gel, gadolinium) allows for more accurate determination of vaginal extension, and it improves the ability to determine the extent of bladder or rectal invasion, if present.[17]

When using MRI for BRT planning as a radiation oncologist, we need to know 1) normal uterine cervix anatomy, 2) cervicouterine junction, and 3) topography and extension of parametrium.[18] The cervix, approximately $2-3 \mathrm{~cm}$ long, is the lowest part of the uterus situated between the endometrial cavity and vagina. It consists of fibromuscular structure and has supravaginal (endocervix) and vaginal (ectocervix) parts. The endocervical canal has high-signal intensity (hyperintense), cervical stroma has low signal intensity (hypointense), and smooth muscle has intermediate signal intensity in non-contrast T2W images. [19] The upper limit of the cervix extends to the uterine corpus like cone shaped, which corresponds to $5 \mathrm{~mm}$ above where the uterine arteries enter the uterus in non-contrast T2W images. On MRI, the parametrium appears as the fat signal intensity and extends anteriorly to the bladder, posteriorly to the perirectal or mesorectal fascia, medially to the tumor or cervical ring, and laterally to the pelvic wall or the medial edge of the internal iliac and obturator veins. Disruption of cervical stromal ring corresponds to the parametrial involvement on MRI (Fig. 1).[19]

Transabdominal US is usually used during the insertion procedure to assist the proper placement of an intrauterine applicator, in particular the suspicion of uterine perforation or in the presence of retrovert or excessive antevert uterus.[20] However, this technique is highly operator dependent. The role of US in contouring and treatment planning are areas of active investigation.[21,22] When used in conjunction with CT-based planning, it has also been shown to be equivalent to MRI-based planning.[23]

The CT scan may also be used in cervical cancer to verify applicator placement, and it ensures that the uterus has not been perforated (Fig. 2). However, soft tissue contrast is poor, and tumor extension is not truly assessed with this technique. Limitations of CT in 3DIGBT can be eliminated by gynecological examination and MRI immediately before BRT.[24-27]
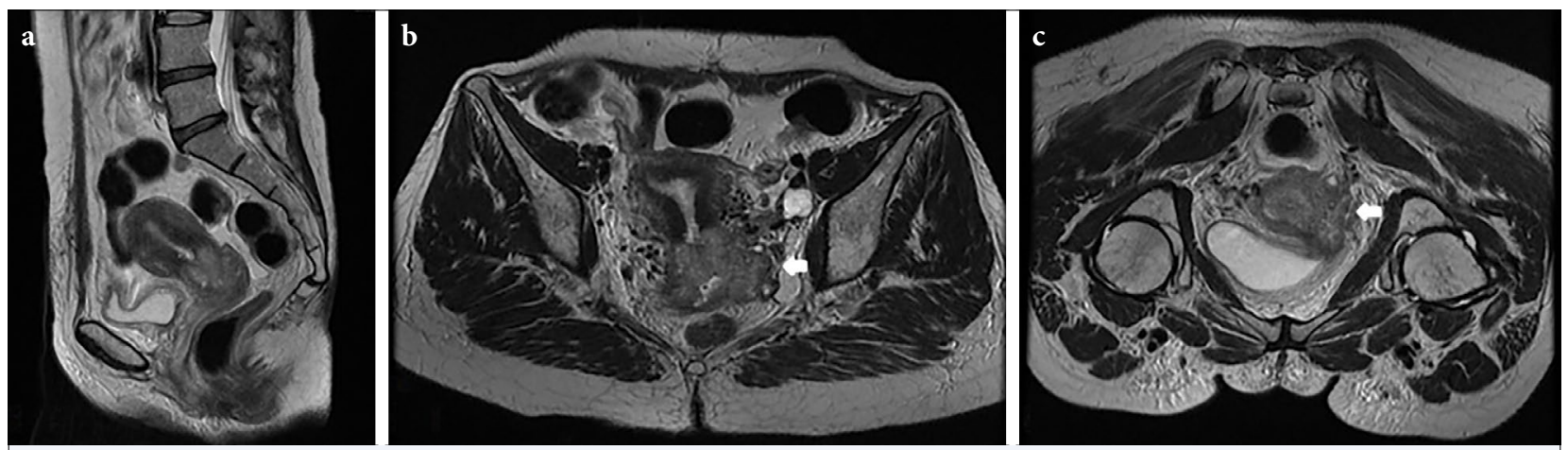

Fig. 1. A patient with a stage IIB cervical cancer with left parametrial involvement (white arrow). Sagittal (a), axial (b), and coronal (c) images of the T2W MRI showing cervical mass with normal uterus. 

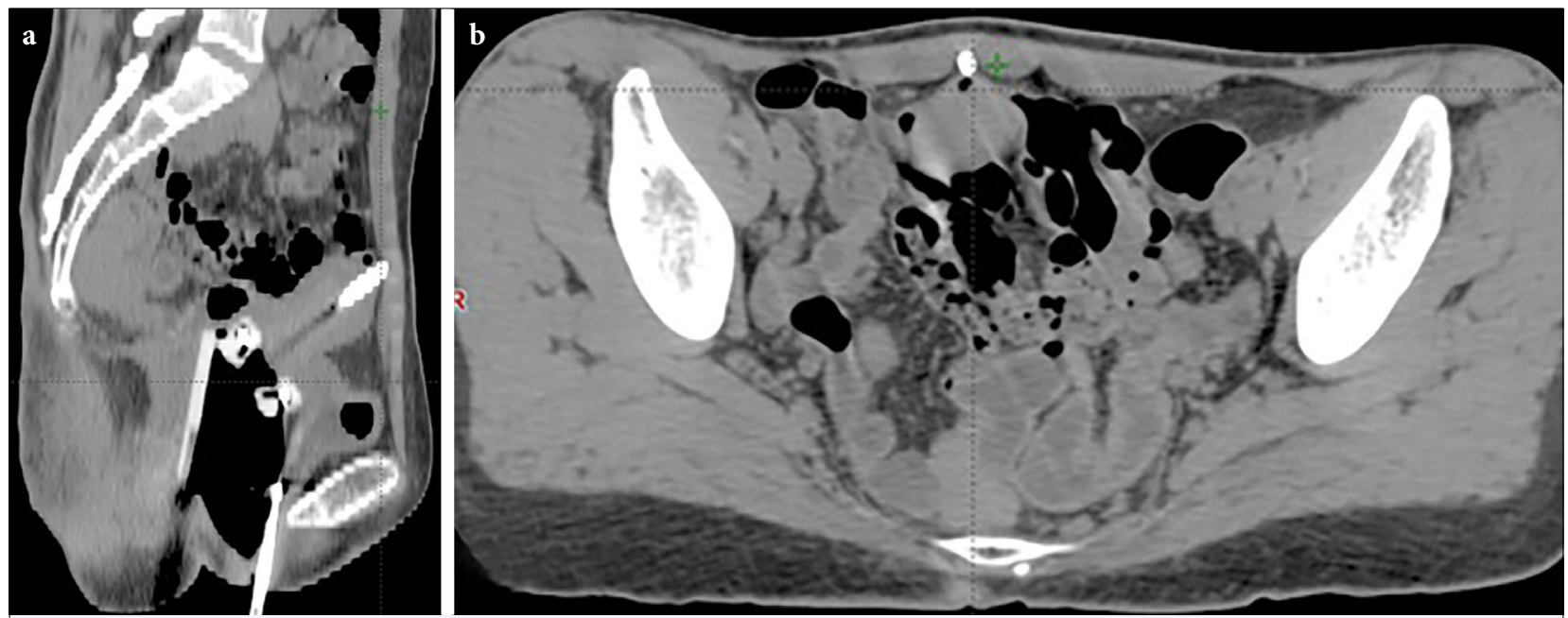

Fig. 2. Sagittal (a) and axial (b) CT images in a 34-year-old woman with stage IB2 cervical cancer show an uterine perforation at first fraction of BRT.
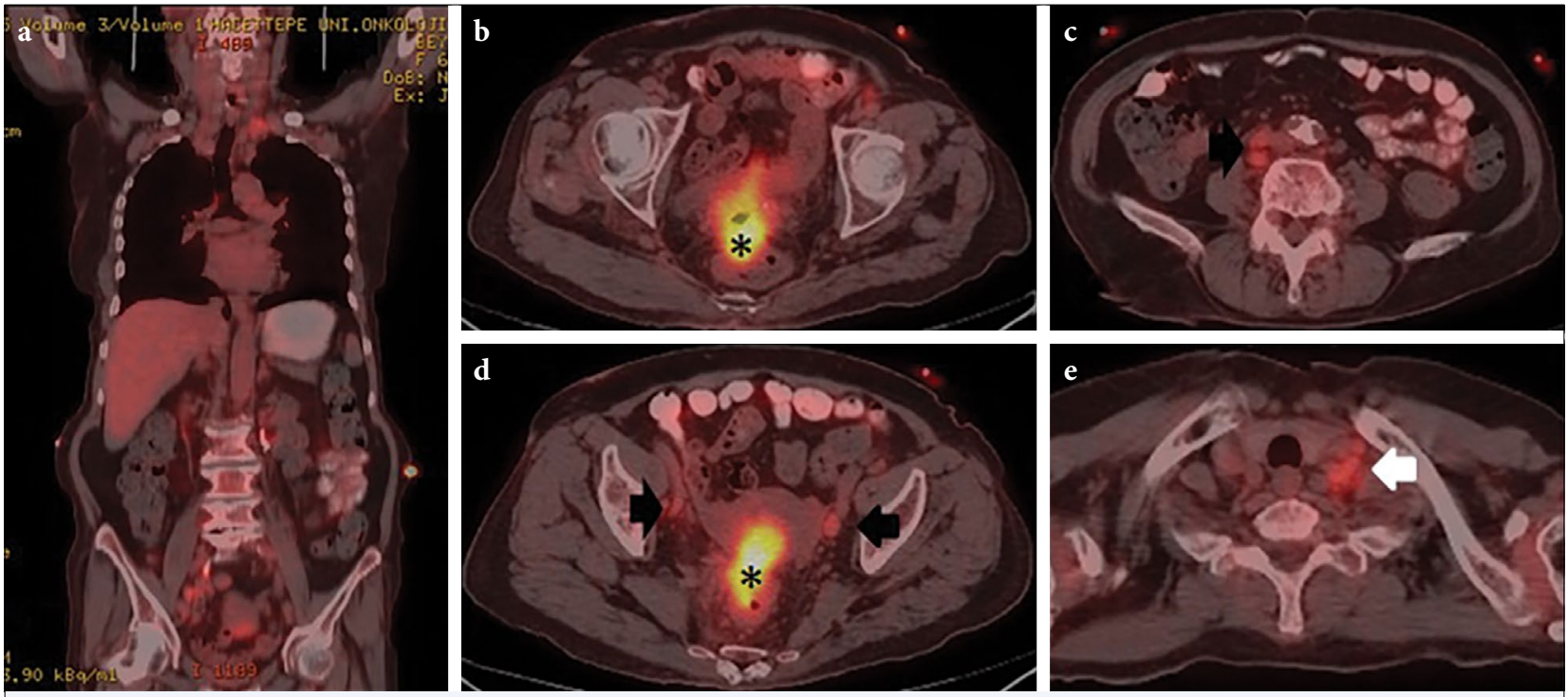

Fig. 3. Coronal (a) and axial (b-e) PET/CT images in a 70-year-old woman with a cervical mass $\left({ }^{*}\right)$ and supraclaviculary (white arrow), pelvic, and para-aortic lymph node metastases (black arrow) at diagnosis.

$\mathrm{PET} / \mathrm{CT}$ is a functional imaging technique that provides metabolic information. It has been widely used in the evaluation of lymph node or distant metastases at diagnosis in cervical cancer (Fig. 3). It can also be used for RT treatment planning, predicting outcome, and assessing treatment response and surveillance.[28]

\section{Target Volume Determination in BRT}

The most important source of uncertainty in the 3DIGBT procedure is related to the target volume delineation with a mean relative standard deviation of $8 \%-$ $10 \%$ for the gross tumor volume (GTV) and high-risk
(HR) clinical target volume (CTV), resulting in cumulative whole-treatment uncertainty of \pm 5 Gy. [29-31] Accurate delineation of target volumes has a direct impact on clinical outcomes, because an inadequate coverage of the GTV and CTV increases the rate of local recurrence.[32]

Target volume determination in 3D-IGBT is performed according to the gynecologic examination and MRI findings at diagnosis and at BRT. The Group Européen de Curiethérapie-European Society for Therapeutic Radiology and Oncology (GEC-ESTRO) recommendations were developed to use a common 
language for 3D-IGBT. [29,33] The first and most important step in target volume assessment is based on clinical examination.[26] As tumor configuration and topography change significantly during EBRT, repetitive gynecological examination is required.[34] GTV, vaginal extension of disease and parametrial involvement should be assessed in every examination, and 3D clinical drawings should be made.

3D-IGBT can be applied in three different ways: 1) MRI can be performed in each BRT fraction with an applicator in place, 2) MRI can be performed with an applicator in place only in the first BRT fraction combined with CT for succeeding fractions, and 3) MRI can be performed before BRT without an applicator in place and fusion can be performed with CT images. Pötter et al. showed that MRI without applicator before BRT had no additional benefit in stage IB tumors but sufficient and useful in limited stage IIB and IIIB cases.[35] In patients with large tumors and severe parametrial involvement, MRI should be performed with applicator.

\section{a. MRI-Based Contouring}

MRI is the gold standard technique for delineation of target volumes in 3D-IGBT. Target volume definitions are made in accordance with the GEC-ESTRO and the International Commission on Radiation Units and Measurements (ICRU) 89 recommendations.[29,33] MRI with T2W sequences is required at diagnosis and at time of BRT with the applicator in place. BRT applicators, which are considerably more expensive than metallic ones, must be MRI compatible (plastic or titanium).

MRI-based assessment of GTV and CTV was to be performed in para-axial, para-sagittal, and paracoronal planes, supplemented by 3D clinical drawings. GTV, CTV, and OARs are contoured according to the clinical examination and MRI at diagnosis and at (each) time of BRT. Primary tumor-GTV includes macroscopic tumor extension at diagnosis (GTVTinit) and at time of BRT (GTV-Tres), as represented by high-signal intensity (hyperintense) masses relative to the healthy cervix on T2W images. CTV includes GTV and subclinical disease. Three CTVs are defined according to tumor load and hence to the risk of recurrence: an HR-CTV with a residual macroscopic disease, an intermediate-risk CTV (IR-CTV) with a residual microscopic disease, and a low-risk CTV (LRCTV) including potential microscopic tumor spread. HR-CTV includes the areas that correspond to major risk of local recurrence at time of each BRT application (HR-CTVB1, HR-CTVB2, etc.). The GTV during $\mathrm{BRT}$, the entire cervix, the extracervical extension and the gray zones in parametria, uterine corpus, vagina or rectum and bladder on MRI are included in HRCTV. IR-CTV (IR-CTVB1, IR-CTVB2, etc.) carrying a significant microscopic tumor load encompasses HRCTV with a safety margin of craniocaudally $1-1.5 \mathrm{~cm}$, anterioposteriorly $0.5 \mathrm{~cm}$, and laterally $1 \mathrm{~cm}$. It is contoured based on macroscopic tumor extension at diagnosis. No safety margin is added if there is no rectal or bladder involvement. No safety margins are added for HR-CTV or PTV.

MRI-based 3D-IGBT requires a high level of experience, and it is often time consuming and expensive. $[29,33]$

\section{b. CT-Based Contouring}

Although MRI with the applicator in place is the "gold standard" technique for 3D-IGBT, MRI units are not available in many radiation oncology department or they are mostly located far from the institution.[36] The CT scans are widely available than MRI, and could be used more frequently for logistic reasons in BRT planning.[25]

For CT-based contouring, the tumor size and topography should routinely be used as a reference on T2W MRI at diagnosis and just before BRT without applicator (Fig. 4). In HR-CTV contouring, all clinical information and MRI findings just before BRT are integrated into the CT images with applicator in place.

To date, there are several guidelines published for CT-based 3D contouring in cervical cancer BRT.
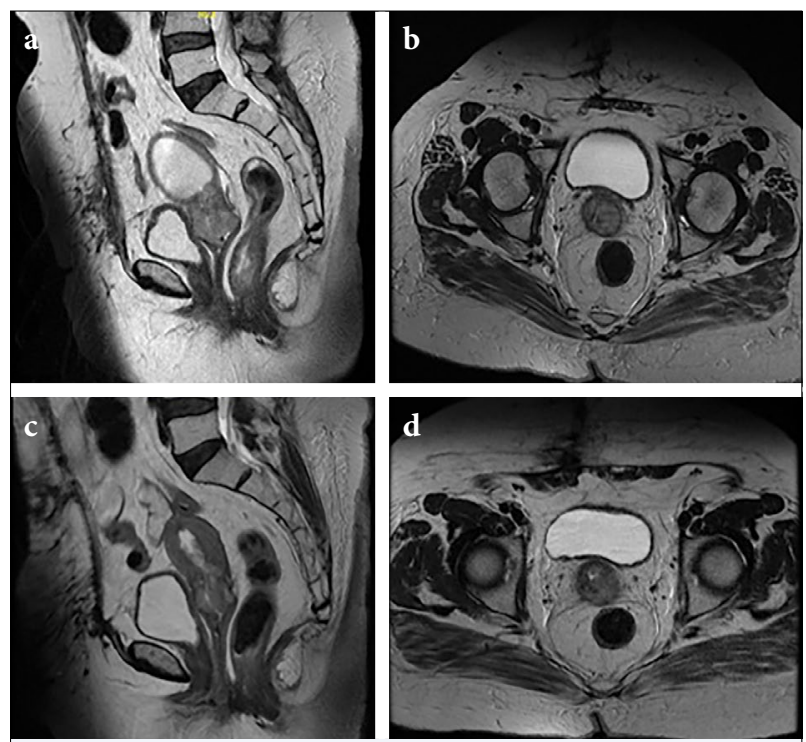

Fig. 4. Sagittal and axial T2W MR images in a 59-yearold woman with stage IIB cervical cancer at diagnosis $(a, b)$ and at the time of BRT $(c, d)$ without applicator. 
[24,37] In 2007, Viswanathan et al. compared the CTbased and MRI-based contours of 10 patients with stage IIA-IIIB cervical cancer to assess the validity of CT-based contours using GEC-ESTRO MRI definitions. [24] All patients underwent both CT and MRI at time of BRT with the tandem and ring applicator in place. On CT-based contouring, the superior border of the cervix and the lateral border of the parametrium were not clearly defined. Also, the cervix and its lateral extension of parametrial tissues were contoured wider than MRI contour. It resulted in a decrease in the D100 and D90. No statistically significant differences were found in the dose for the OARs.

In 2014, Viswanathan et al. compared the CT-based and MRI-based contours in local advanced cervical cancers, and generated a 95\% consensus volume.[37] Online contouring atlases for 3D-IGBT are available for instruction at http://www.nrgoncology.org/Resources/ ContouringAtlases.aspx. In this study, 23 gynecologic radiation oncology expert from the Radiation Therapy Oncology Group contoured same three cervical cancer cases: stage IIB, near-complete response; stage IIB, partial response; and stage IB2, complete response. All patients had a 3T MRI at diagnosis, an MRI and a CT performed at the time of BRT (within an hour of applicator insertion), and clinical drawings. When CT and MRI volumes were compared, the mean tumor volume was larger on CT than on MRI for all three cases. Among physicians' contours, CT had a higher level of agreement. There was no statistically significant difference in D90 or D2cc OARs comparing CT to MRI. The lowest concordance between CT and MRI contours was found for a patient with a stage IIB cervical cancer with a near-complete response to chemoradiotherapy. The highest concordance between CT and MRI contours was found for a patient with a stage IB2 cervical cancer with a complete response. The concordance between CT and MRI contours was good in a patient with a stage IIB disease with a partial response. According to this study, patients with no parametrial extension at diagnosis and with a good response to EBRT are least likely to benefit from the use of MRI. On the contrary, patients with a large tumor at diagnosis with parametrial extension and with a near-complete response are most likely to benefit from the use of MRI.

In 2017, Ohno et al. published recommendations for contouring the CT-based HR-CTV for 3D-IGBT for cervical cancers. [38] In this study, 15 gynecologic radiation oncology experts from the Japanese Radiation Oncology Study Group defined CT-based HR-CTV boundaries in cranial-caudal, lateral, or anterior-posterior planes. To minimize the difference in width between CT-based HR-CTV and MRI-based HR-CTV, they recommended to 1) reduce the slice thickness to $<3 \mathrm{~mm}, 2$ ) determine the lateral border carefully, and 3) exclude the visible linear structures that run laterally (e.g. the vessels, nerves and non-tumor fibrous structures).

All these HR-CTV contouring guidelines for CT are summarized in Table 1.

The superior extent of the cervix cannot be clearly determined by CT. However, it encompasses the average cervical height of $3 \mathrm{~cm}$. If intravenous (IV) contrast material is given, the superior extent of the cervix corresponds to the cervicouterine junction where the uterine vessels abut to the cervix. It can be delineated as the superior border of the HR-CTV. MRI immediately before or at BRT may help to accurately determine the superior border of the HR-CTV. If MRI is not available, HR-CTV should include a minimum two-thirds of the uterine height.[26] It is also critical to avoid unnecessarily contouring uninvolved parametrial tissue. The inferior border of the HR-CTV is more accurately delineated based on gynecologic examination with 3D clinical drawings at diagnosis and at BRT. On CT, borders of tumor, cervix, and parametrium could not be distinguished; and GTV cannot be delineated. HRCTV includes the entire cervix and any notable residual tissue at parametrium, uterus, vagina, rectum, and bladder. If adjacent organ invasion is present, region of tumor invasion into adjacent organ should be contoured. IV contrast is not mandated. However, when the contrast material is given to the bladder or rectum, the cervix can be more clearly defined. When IR-CTV is contouring, $1 \mathrm{~cm}$ safety margin is added around

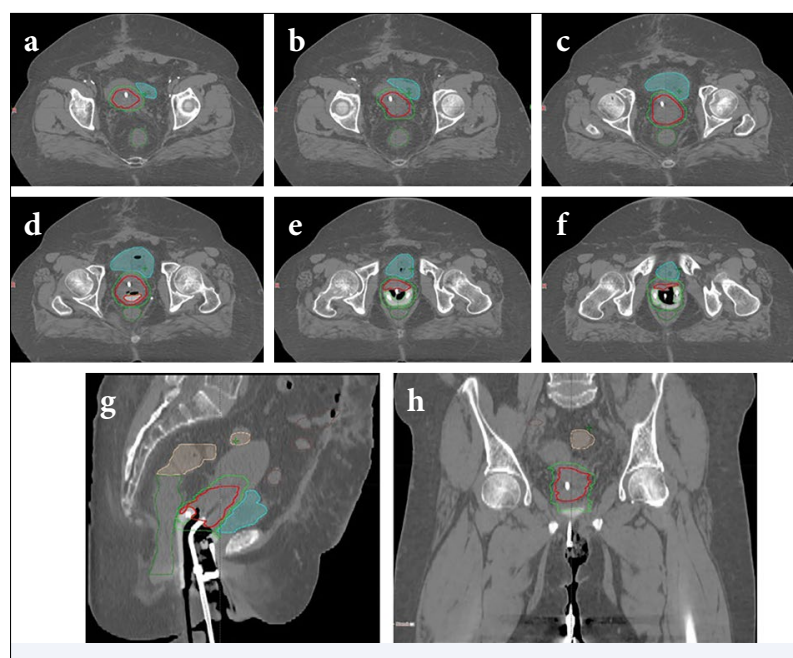

Fig. 5. CT-based HR-CTV and IR-CTV volumes in a same patient with residual disease at time of BRT. Axial (a-f), sagittal (g), and coronal (h) images. Red line $=H R-C R V$, green line $=I R-C T V$, cyan=bladder, dark green $=$ rectum, pink=sigmoid. 
Table 1 HR-CTV contouring guidelines for CT

Viswanathan AN et al. (2007)

Inferior Upper level of the applicator or lowest part of the vaginal extension
Viswanathan AN et al. $(2014)^{37}$

Starts from ring or ovoid level

Tissue inside the central ring or to the level of the ovoids should be contoured

If there is an involvement at the time of BRT, vaginal tissue adjacent to the ring should be contoured

If IV contrast is given, the level where the uterine vessels abut the cervix or where the uterine tissue/cavity begins

Superior Additional two slices are contoured around tandem superiorly with decreasing diameter (to include conical cervical apex)

Cervical height should be measured $(\sim 3 \mathrm{~cm})$

If inner half of the parametrium is involved laterally contour $\leq 2 \mathrm{~cm}$ from edge of cervix

Lateral If outer half of the parametrium is involved laterally contour $>2 \mathrm{~cm}$ from edge of cervix

Parametrial extension (gray/ white on the $\mathrm{CT}$ ) should be included (similar density to the cervix)
The level where the uterus begins (internal os), contour the next 1 $\mathrm{cm}$ as a cone shaped

Cervical height $\sim 3 \mathrm{~cm}$

\section{Ohno T et al. $(2017)^{38}$}

Cervical tissue at the level of the tandem applicator fringe

Contour exophytic tumors extending to the vaginal cavity at the time of BRT

Applicators, vaginal packing, and vaginal vault are not included

If vaginal invasion at diagnosis, residual vaginal tumor at the time of BRT and entire vaginal wall should be contoured

Starts at the junction of the uterine artery or serosal side of the uterine isthmus, contour the next $1 \mathrm{~cm}$ as a cone shaped

If uterine corpus invasion at diagnosis, abnormal signal intensity (gray zone) on MRI just before BRT should be contoured

Border between the uterine tissue or residual tumor (soft tissue density on $\mathrm{CT}$ ) and surrounding adipose tissue (low density on (T) at the time of BRT

Bowel, adnexa, ascites, and visible linear structures (e.g. vessels, nerves and fibrous tissues) that run laterally are not included.

Calcifications at the periphery of the uterus can determine the lateral border

Border between the uterine tissue or residual tumor at the time of $\mathrm{BRT}$ and the adipose tissue

If there is no adipose tissue, bladder wall is not included

If there is an invasion to the bladder wall at the time of BRT, residual bladder invasion should be contoured

For invasion of the bladder, muscle layer invasion should be confirmed

Border between the uterine tissue or residual tumor at the time of $\mathrm{BRT}$ and the adipose tissue

If there is no adipose tissue, the walls of the rectum, sigmoid colon, and small bowels are not included

Involved rectum or sigmoid colon walls at the time of BRT should be contoured

For invasion of the rectum or sigmoid colon wall, muscle layer invasion should be confirmed 
HR-CTV, and it is modified by tumor extension at diagnosis. IR-CTV should include the parametrial, uterosacral, and vaginal disease at diagnosis. If there is no involvement, the contour should not extend to the bladder, sigmoid, rectum, and pelvic bones. Figure 5 shows CT-based target volumes.

\section{Conclusion}

We conclude that contouring guidelines should be considered in 3D-IGBT for cervical cancers. The MRIbased BRT with applicator in place is the gold standard technique, especially in patients with large tumors and parametrial involvement. The CT scans are adequate for OARs delineation, but the cervix cannot be assessed clearly and CT-based target contours significantly wider than with MRI. Gynecological examination and MRI immediately before BRT can eliminate limitations of CT. It should be kept in mind that 3D-IGBT requires considerable time and high level of experience.

Peer-review: Externally peer-reviewed.

Conflict of Interest: None declared.

Financial Support: None declared.

\section{References}

1. Rose PG, Bundy BN, Watkins EB, Thigpen JT, Deppe G, Maiman MA, et al. Concurrent cisplatin-based radiotherapy and chemotherapy for locally advanced cervical cancer. N Engl J Med 1999;340(15):1144-53.

2. Thomas GM. Improved treatment for cervical cancerconcurrent chemotherapy and radiotherapy. N Engl J Med 1999;340(15):1198-200.

3. Stehman FB, Ali S, Keys HM, Muderspach LI, Chafe WE, Gallup DG, et al. Radiation therapy with or without weekly cisplatin for bulky stage $1 \mathrm{~B}$ cervical carcinoma: follow-up of a Gynecologic Oncology Group trial. Am J ObstetGynecol . 2007;197(5):503.e1-6.

4. Mazeron R, Castelnau-Marchand P, Dumas I, del Campo ER, Kom LK, Martinetti F, et al. Impact of treatment time and dose escalation on local control in locally advanced cervical cancer treated by chemoradiation and image-guided pulsed-dose rate adaptive brachytherapy. Radiother Oncol 2015;114(2):257-63.

5. Han K, Milosevic M, Fyles A, Pintilie M, Viswanathan AN. Trends in the utilization of brachytherapy in cervical cancer in the United States. Int J Radiat Oncol Biol Phys 2013;87(1):111-9.

6. Pötter R, Georg P, Dimopoulos JC, Grimm M, Berger D, Nesvacil N, et al. Clinical outcome of protocol based image (MRI) guided adaptive brachytherapy combined with 3D conformal radiotherapy with or without chemotherapy in patients with locally advanced cervical cancer. Radiother Oncol 2011;100(1):116-23.

7. Charra-Brunaud C, Harter V, Delannes M, HaieMeder C, Quetin P, Kerr C, et al. Impact of 3D image-based PDR brachytherapy on outcome of patients treated for cervix carcinoma in France: results of the French STIC prospective study. Radiother Oncol 2012;103(3):305-13.

8. Ribeiro I, Janssen H, De Brabandere M, Nulens A, De Bal D, Vergote I, et al. Long term experience with 3D image guided brachytherapy and clinical outcome in cervical cancer patients. Radiother Oncol 2016;120(3):447-54.

9. Sturdza A, Pötter R, Fokdal LU, Haie-Meder C, Tan LT, Mazeron R, et al. Image guided brachytherapy in locally advanced cervical cancer: Improved pelvic control and survival in RetroEMBRACE, a multicenter cohort study. Radiother Oncol 2016;120(3):428-33.

10. Tanderup K, Fokdal LU, Sturdza A, Haie-Meder C, Mazeron R, van Limbergen E, et al. Effect of tumor dose, volume and overall treatment time on local control after radiochemotherapy including MRI guided brachytherapy of locally advanced cervical cancer. Radiother Oncol 2016;120(3):441-6.

11. Pötter R, Dimopoulos J, Georg P, Lang S, Waldhäusl C, Wachter-Gerstner N, et al. Clinical impact of MRI assisted dose volume adaptation and dose escalation in brachytherapy of locally advanced cervix cancer. Radiother Oncol 2007;83(2):148-55.

12. Lindegaard JC, Fokdal LU, Nielsen SK, Juul-Christensen J, Tanderup K. MRI-guided adaptive radiotherapy in locally advanced cervical cancer from a Nordic perspective. Acta Oncol 2013;52(7):1510-9.

13. Dimopoulos JC, Petrow P, Tanderup K, Petric P, Berger D, Kirisits C, et al. Recommendations from Gynaecological (GYN) GEC-ESTRO Working Group (IV): Basic principles and parameters for MR imaging within the frame of image based adaptive cervix cancer brachytherapy. Radiother Oncol 2012;103(1):113-22.

14. Tanderup K, Viswanathan AN, Kirisits C, Frank SJ. Magnetic resonance image guided brachytherapy. Semin Radiat Oncol 2014;24(3):181-91.

15. Haack S, Pedersen EM, Jespersen SN, Kallehauge JF, Lindegaard JC, Tanderup K. Apparent diffusion coefficients in GEC ESTRO target volumes for image guided adaptive brachytherapy of locally advanced cervical cancer. Acta Oncol 2010;49(7):978-83.

16. Esthappan J, Ma DJ, Narra VR, Raptis CA, Grigsby PW. Comparison of apparent diffusion coefficient 
maps to T2-weighted images for target delineation in cervix cancer brachytherapy. J Contemp Brachytherapy 2011;3(4):193-8.

17. Robbins JB, Sadowski EA, Jolly S, Maturen KE. MR Imaging in Gynecologic Brachytherapy. Magn Reson Imaging Clin N Am 2017;25(3):651-66.

18. Petric P, Mohammed-Al-Hammadi N. MRI findings at image guided adaptive cervix cancer brachytherapy: radiation oncologist's perspective. J Contemp Brachytherapy 2014;6(2):215-22.

19. Sullivan T, Yacoub JH, Harkenrider MM, Small W Jr, Surucu M, Shea SM. Providing MR Imaging for Cervical Cancer Brachytherapy: Lessons for Radiologists. Radiographics 2018;38(3):932-44.

20. Davidson MT, Yuen J, D'Souza DP, Radwan JS, Hammond JA, Batchelar DL. Optimization of high-doserate cervix brachytherapy applicator placement: the benefits of intraoperative ultrasound guidance. Brachytherapy 2008;7(3):248-53.

21. St-Amant P, Foster W, Froment MA, Aubin S, Lavallée MC, Beaulieu L. Use of 3D transabdominal ultrasound imaging for treatment planning in cervical cancer brachytherapy: Comparison to magnetic resonance and computed tomography. Brachytherapy 2017;16(4):847-54.

22. Tharavichitkul E, Chakrabandhu S, Klunklin P, Onchan W, Jia-Mahasap B, Wanwilairat S, et al. Intermediate-term results of trans-abdominal ultrasound (TAUS)-guided brachytherapy in cervical cancer. Gynecol Oncol 2018;148(3):468-73.

23. Mahantshetty U, Naga Ch P, Khadanga CR, Gudi S, Chopra S, et al. A Prospective Comparison of Computed Tomography with Transrectal Ultrasonography Assistance and Magnetic Resonance Imaging-Based Target-Volume Definition During Image Guided Adaptive Brachytherapy for Cervical Cancers. Int J Radiat Oncol Biol Phys 2018. pii: S0360-3016(18)30933-7.

24. Viswanathan AN, Dimopoulos J, Kirisits C, Berger D, Pötter R. Computed tomography versus magnetic resonance imaging-based contouring in cervical cancer brachytherapy: results of a prospective trial and preliminary guidelines for standardized contours. Int J Radiat Oncol Biol Phys 2007;68(2):491-8.

25. Viswanathan AN, Creutzberg CL, Craighead P, McCormack M, Toita T, Narayan K, et al. International brachytherapy practice patterns: a survey of the Gynecologic Cancer Intergroup (GCIG). Int J Radiat Oncol Biol Phys 2012;82(1):250-5.

26. Hegazy N, Pötter R, Kirisits C, Berger D, Federico $\mathrm{M}$, Sturdza A, et al. High-risk clinical target volume delineation in CT-guided cervical cancer brachyther- apy: impact of information from FIGO stage with or without systematic inclusion of 3D documentation of clinical gynecological examination. Acta Oncol 2013;52(7):1345-52.

27. Nesvacil N, Pötter R, Sturdza A, Hegazy N, Federico M, Kirisits C. Adaptive image guided brachytherapy for cervical cancer: a combined MRI-/CT-planning technique with MRI only at first fraction. Radiother Oncol 2013;107(1):75-81.

28. Herrera FG, Prior JO. The role of PET/CT in cervical cancer. Front Oncol 2013;3:34.

29. Haie-Meder C, Pötter R, Van Limbergen E, Briot E, De Brabandere M, Dimopoulos J, et al. Recommendations from Gynaecological (GYN) GEC-ESTRO Working Group (I): concepts and terms in 3D image based 3D treatment planning in cervix cancer brachytherapy with emphasis on MRI assessment of GTV and CTV. Radiother Oncol 2005;74(3):235-45.

30. Tanderup K, Nesvacil N, Pötter R, Kirisits C. Uncertainties in image guided adaptive cervix cancer brachytherapy: impact on planning and prescription. Radiother Oncol 2013;107(1):1-5.

31. Hellebust TP, Tanderup K, Lervåg C, Fidarova E, Berger D, Malinen E, et al. Dosimetric impact of interobserver variability in MRI-based delineation for cervical cancer brachytherapy. Radiother Oncol 2013;107(1):13-9.

32. Eifel PJ, Thoms WW Jr, Smith TL, Morris M, Oswald MJ. The relationship between brachytherapy dose and outcome in patients with bulky endocervical tumors treated with radiation alone. Int J Radiat Oncol Biol Phys 1994;28(1):113-8.

33. Pötter R, Haie-Meder C, Van Limbergen E, Barillot I, De Brabandere M, Dimopoulos J, et al. Recommendations from gynaecological (GYN) GEC ESTRO working group (II): concepts and terms in 3D image-based treatment planning in cervix cancer brachytherapy$3 \mathrm{D}$ dose volume parameters and aspects of $3 \mathrm{D}$ image-based anatomy, radiation physics, radiobiology. Radiother Oncol 2006;78(1):67-77.

34. Mayr NA, Taoka T, Yuh WT, Denning LM, Zhen WK, Paulino AC, et al. Method and timing of tumor volume measurement for outcome prediction in cervical cancer using magnetic resonance imaging. Int $J$ Radiat Oncol Biol Phys 2002;52(1):14-22.

35. Pötter R, Federico M, Sturdza A, Fotina I, Hegazy N, Schmid M, et al. Value of Magnetic Resonance Imaging Without or With Applicator in Place for Target Definition in Cervix Cancer Brachytherapy. Int J Radiat Oncol Biol Phys 2016;94(3):588-97.

36. Dimopoulos JC, Schard G, Berger D, Lang S, Goldner G, Helbich T, et al. Systematic evaluation of MRI 
findings in different stages of treatment of cervical cancer: potential of MRI on delineation of target, pathoanatomic structures, and organs at risk. Int J Radiat Oncol Biol Phys 2006;64(5):1380-8.

37. Viswanathan AN, Erickson B, Gaffney DK, Beriwal S, Bhatia SK, Lee Burnett O 3rd, et al. Comparison and consensus guidelines for delineation of clinical target volume for CT- and MR-based brachytherapy in lo- cally advanced cervical cancer. Int J Radiat Oncol Biol Phys 2014;90(2):320-8.

38. Ohno T, Wakatsuki M, Toita T, Kaneyasu Y, Yoshida $\mathrm{K}$, Kato S, et al. Recommendations for high-risk clinical target volume definition with computed tomography for three-dimensional image-guided brachytherapy in cervical cancer patients. J Radiat Res 2017;58(3):341-50. 